\title{
Early salvage radiation therapy combined with short-term hormonal therapy in recurrent prostate cancer after radical prostatectomy: Single-institution 4-year data on outcome, toxicity, health-related quality of life and co-morbidities from 184 consecutive patients treated with 70 Gy
}

\author{
JEFF R. CORTÉS-GONZÁLEZ ${ }^{1,2}$, ENRIQUE CASTELLANOS ${ }^{1}$, KATINKA SANDBERG ${ }^{1}$, \\ MARIE-HJELM ERIKSSON $^{1}$, PETER WIKLUND ${ }^{3}$, STEFAN CARLSSON ${ }^{3}$, GABRIELLA COHN-CEDERMARK $^{1}$, \\ ULRIKA HARMENBERG $^{1}$, OVE GUSTAFSSON ${ }^{4}$, SEYMOUR H. LEVITT $^{1,5}$, \\ BO LENNERNÄS ${ }^{1,6}$, YVONNE BRANDBERG $^{1}$, MARCELA MÁRQUEZ ${ }^{1}$, \\ KARL-MIKAEL KÄLKNER ${ }^{1}$ and STEN NILSSON ${ }^{1}$
}

\begin{abstract}
${ }^{1}$ Department of Oncology-Pathology, Karolinska Institutet, Karolinska University Hospital, Stockholm, Sweden;
${ }^{2}$ Urology Service, University Hospital ‘Dr. Jose E. Gonzalez’ UANL, Monterrey, Mexico; ${ }^{3}$ Department of Surgery, Section of Urology, Karolinska University Hospital, Stockholm and Section of Urology, Department of Molecular Medicine and Surgery, Karolinska Institutet; ${ }^{4}$ Division of Urology, Department of Clinical Science, Intervention and Technology,

Karolinska Institutet, Karolinska University Hospital, Stockholm, Sweden; ${ }^{5}$ Department of Radiation Oncology, University of Minnesota, Minneapolis, MN, USA; ${ }^{6}$ Department of Oncology, University of Gothenburg, Sahlgrenska University Hospital, Gothenburg Urology Service, Gothenburg, Sweden
\end{abstract}

Received April 13, 2012; Accepted June 21, 2012

DOI: 10.3892/ijo.2012.1694

\begin{abstract}
The aim of this study was to investigate the role of 70 Gy salvage radiotherapy (SRT) combined with shortterm neoadjuvant hormonal therapy (NHT) in the treatment of recurrent disease after radical prostatectomy (RP), and to consider quality of life $(\mathrm{QoL})$, survival outcomes and impact of co-morbidities on treatment-related rectal-genitourinary toxicity. Electronic records of 184 SRT patients treated consecutively between October 2001 and February 2007 were analyzed. Median age was 64 years (median follow-up 48 months). NHT was given to 165 patients (median 3 months). Pre-RP and pre-SRT PSA, PSA doubling time, Gleason score (GS), seminal vesicle invasion (SVI) and detectable post-SRT PSA were recorded. Any detectable PSA or PSA $>0.1 \mathrm{ng} / \mathrm{ml}+$ nadir was considered biochemical failure $(\mathrm{BcF})$. The Charlson co-morbidity index was used to correlate co-morbidities and rectal-genitourinary toxicity. Scores from the health-related QoL EORTC QLQ-C30 and PR-25 questionnaires were also evaluated. In $116(63 \%)$ patients, a long-lasting curative
\end{abstract}

\footnotetext{
Correspondence to: Dr Jeff R. Cortés-González, Department of Oncology-Pathology, Karolinska Institutet, Urologic Oncology Group CCK R8:3, SE-17176 Stockholm, Sweden

E-mail: jeff.cortes-gonzalez@ki.se
}

Key words: prostate cancer, salvage radiotherapy, radical prostatectomy, neoadjuvant hormone therapy, PSA, co-morbidity, quality of life effect was indicated by undetectable PSA levels. In univariate analysis, using $\mathrm{BcF}$ as an outcome variable, $\mathrm{p}<0.001$ was found for GS, pre-SRT PSA, SVI and detectable post-SRT PSA. Multivariate analysis showed $\mathrm{p}=0.01$ for SVI, $\mathrm{p}=0.09$ for $\mathrm{GS}$, and detectable post-SRT PSA ( $\mathrm{p}=0.01)$; with metastases as an outcome variable, only SVI was significant $(\mathrm{p}=0.007)$. Cancerspecific and overall survival were 99 and 95\%, respectively. Although microscopy showed SVI or GS 8-10 in the prostatectomy specimens $17 / 40(43 \%)$ and $13 / 29(45 \%)$, respectively, of patients still showed undetectable PSA at long-term follow-up (median 55 months) after SRT. Likewise, 11/31 (36\%) patients with pre-SRT PSA $>1.0 \mathrm{ng} / \mathrm{ml}$ and 80/134 (60\%) patients with PSA doubling time (PSADT) $<10$ still showed undetectable PSA after 50 months. Slightly elevated acute and late rectalgenitourinary grade 3-4 toxicity was observed. No association with co-morbidity/toxicity was found. EORTC QLQ-C30 scores were similar to or slightly better than reference values. SRT with 70 Gy combined with 3-month NHT results in long-term undetectable PSA in $>50 \%$ of patients with recurrence after RP with acceptable rectal-genitourinary toxicity and without negatively affecting long-term QoL. Non-metastatic patients should not be disqualified from receiving SRT although presenting with poor prognostic factors at surgery.

\section{Introduction}

Every third patient with localized prostate cancer (PCa) who undergoes radical prostatectomy (RP) will experience 
biochemical failure $(\mathrm{BcF})$ within 10 years $(1,2)$ and, without further treatment, one third will progress to metastatic disease within 8 years (1). Postoperative radiotherapy has been delivered to patients with recurrent PCa for the last 2-3 decades, but not until recently have data shown that adjuvant radiotherapy (ART) increases disease-free survival and also provides an appreciable survival benefit in patients with non-radically excised tumors (3-5). Definitions of BcF have varied over the years, although they have all included rising PSA after RP and no evidence of clinical or radiological residual disease. Furthermore, there is now level 1 evidence that ART plays a role after non-radical $\mathrm{RP}$, but the vast majority of patients with PSA relapse have not received this treatment.

Thus, therapy decisions must be made as to whether these individuals should be offered salvage radiotherapy (SRT). Several ongoing studies include patients randomized to ART or SRT; however, data are not to be expected within the next few years, and, hence, decisions must be based on outcome data from non-randomized studies. Numerous questions concerning SRT remain to be answered, although recommendations and guidelines have emerged $(6,7)$. Common to these is that SRT provides long-term control of the disease in approximately one third of patients with BcF, particularly those with relatively low pre-SRT PSA levels. Patients with poorly differentiated cancer and short PSA doubling time (PSADT) seem to benefit most from SRT (8). Recently, well-designed matched-pair analyses were performed to compare ART with early SRT $(9,10)$ and these investigations, like the three large randomized adjuvant trials, suggested that ART is superior. However, it is difficult to draw extensive conclusions from the indicated observations, because, by definition, the SRT patients suffered from more advanced disease. Treatment decisions can be based on previously reported outcome data. However, knowledge is still limited regarding issues such as long-term side-effects, impact of treatment on health-related quality of life (HRQoL), influence of co-morbidity on treatment outcome, effects of neoadjuvant hormone therapy (NHT).

The aim of the present study was to evaluate the long-term outcome of SRT, with consideration paid to genitourinary (GU) and gastrointestinal (GI) toxicity, the impact on HRQoL, and the influence of co-morbidity. To our knowledge, this is the first large, single-institution study to use a uniform radiation treatment protocol combined with short-term NHT.

\section{Patients and methods}

Patients. Treatment outcome was analyzed in 184 consecutive PCa patients with post-RP BcF. The patients had been referred for SRT to the Radiumhemmet and Södersjukhuset facilities at the Karolinska University Hospital (KS) in Stockholm, Sweden, between October 2001 and February 2007. Patients were available for all the following characteristics: pT stage 2-3, record of digital rectal examination (DRE), post-RP BcF, NX or N0, Gleason score (GS) of the post-RP specimen and at least 12 months post-SRT follow-up. Median age was 64 years (range 39-77), and median follow-up was 48 months (range 12-92). After SRT, all patients were monitored for $\mathrm{BcF}$ with periodic PSA values. If recurrence/metastases were suspected, scintigraphy and/or $\left[\mathrm{F}^{18}\right]$-FDG or acetate positron emission tomography were performed.
Table I. Baseline clinical characteristics.

\begin{tabular}{ll}
\hline Characteristics & No. $(\%)^{\mathrm{a}}$ \\
\hline Total no. of patients & 184 \\
& \\
$\begin{array}{l}\text { Pre-prostatectomy age, median } \\
\text { (range), ng/ml }\end{array}$ & $64(39-77)$ \\
& \\
Pre-prostatectomy PSA level, median & \\
(range), ng/ml & $9.5(1.8-48)$ \\
$\quad$ PSA $<10$ & \\
PSA $10-20$ & $101(55)$ \\
PSA $>20$ & $68(37)$ \\
Pathological stage & $15(8)$ \\
pT2 & \\
pT3 & $63(34)$ \\
\end{tabular}
Gleason score after radical prostatectomy $\leq 6$
7
$8-10$
$30(16)$

Digital rectal examination

Positive

$46(25)$

Biopsy

$35(76)$

Positive

Pre-radiotherapy PSA level, median

$0.47(0.1-6.3)$

(range), $\mathrm{ng} / \mathrm{ml}$

PSA doubling time, median (range), months

$6(0.4-78)$

PSA doubling time $<10$ months

Positive surgical margins

Seminal vesicle invasion

$40(22)$

Disease-free interval between prostatectomy and radiotherapy, median (range), months

Persistent detectable PSA level after radical $85(46)$ prostatectomy

Neoadjuvant androgen deprivation therapy $165(90)$ before salvage radiotherapy

$\begin{array}{ll}\text { Radiation dose } & 70 \mathrm{~Gy} \\ \text { Entire seminal vesicles } & 78(43) \\ \text { Shrinking field after 54 Gy } & 87(47) \\ \text { Only prostate bed } & 19(10)\end{array}$

Follow-up after radiotherapy, median

$48(12-92)$ (range), months

PSA, prostate-specific antigen. ${ }^{a}$ Except where otherwise indicated. 
Table II. Association of rectal (GI)/genitourinary (GU) toxicity and comorbidities based on the RTOG toxicity score and the Charlson co-morbidity index adjusted for age (CCIAA) at different time-points.

\begin{tabular}{|c|c|c|c|c|c|c|c|}
\hline GU RTOG score & $\begin{array}{c}\text { CCIAA } \\
\text { L/M n }(\%)\end{array}$ & $\begin{array}{c}\text { CCIAA } \\
\text { High n }(\%)\end{array}$ & p-value & GI RTOG score & $\begin{array}{c}\text { CCIAA } \\
\mathrm{L} / \mathrm{M} \text { n }(\%)\end{array}$ & $\begin{array}{c}\text { CCIAA } \\
\text { High n }(\%)\end{array}$ & p-value \\
\hline Acute 0 & $48(42)$ & $31(45)$ & 0.75 & Acute 0 & $28(24)$ & $24(35)$ & 0.13 \\
\hline Acute 1-2 & $65(57)$ & $36(52)$ & 0.64 & Acute 1-2 & $87(76)$ & $45(65)$ & 0.13 \\
\hline Acute 3-4 & $2(2)$ & $2(3)$ & 0.63 & Acute 3-4 & 0 & 0 & \\
\hline At 2 years 0 & $89(77)$ & $42(61)$ & 0.01 & At 2 years 0 & $88(77)$ & $52(75)$ & 0.86 \\
\hline At 2 years $1-2$ & $24(21)$ & $24(35)$ & 0.06 & At 2 years $1-2$ & $27(23)$ & $15(22)$ & 0.85 \\
\hline At 2 years $3-4$ & $2(2)$ & $3(4)$ & 0.36 & At 2 years 3-4 & $0(0)$ & $2(3)$ & 0.13 \\
\hline At 5 years 0 & $48(72)$ & $27(60)$ & 0.22 & At 5 years 0 & $56(86)$ & $39(87)$ & 1.00 \\
\hline At 5 years $1-2$ & $13(19)$ & $13(29)$ & 0.26 & At 5 years $1-2$ & $6(9)$ & $4(9)$ & 1.00 \\
\hline At 5 years $3-4$ & $6(9)$ & $5(11)$ & 0.75 & At 5 years 3-4 & $3(5)$ & $2(4)$ & 1.00 \\
\hline
\end{tabular}

GU/GI toxicity was graded 0-4 (RTOG/EORTC scale) (11). For further comparisons, patients were divided into three subgroups, with grades $0,1-2$, and 3-4, respectively. Prevalence of the highest toxicity score during different time frames was recorded. Toxicity was considered to be late if it occurred at $>6$ months after SRT, and acute if it arose earlier. Any post-RP GU symptoms were recorded only if they had become worse after SRT. The Charlson co-morbidity index (CCI) evaluates 19 conditions (12). To calculate the CCI, a weight to each disease category is assigned, based on all discharge diagnoses (KS database) that were present before and/or during the SRT. The cancer variable was weighted when a second malignancy was present (13). To adjust for age, each decade (starting at 50 years) was counted as an extra point. Weights were summed, and a total score was obtained (12). Two subgroups were delineated for further comparisons: $0-2$ and $\geq 3$ as low/medium and high scores, respectively. L/M, low-medium score.

Treatment. Radical prostatectomy was retropubic in $161(87 \%)$, laparoscopic in $3(2 \%)$, and robot-assisted in $20(11 \%)$. Three different clinical target radiotherapy volumes (CTVs) were used according to individual factors: A) inclusion of the prostatic fossa (PF) and seminal vesicles (SVs) in the $78(43 \%)$ patients with SV invasion (SVI) at surgery; B) inclusion of only the PF in $19(10 \%)$ patients whose SVs had been removed completely; C) a shrinking field in $87(47 \%)$ patients with residual distal parts of the SVs after RP. The planning target volume (PTV) was defined as CTV plus $20 \mathrm{~mm}$ in all directions except posteriorly, where $15 \mathrm{~mm}$ was used. The daily fraction was 2 Gy delivered 5 days/week. For CTVs A and B, the prescribed dose was $70 \mathrm{~Gy}$; in $\mathrm{C}$, the PF and the SVs were irradiated to $54 \mathrm{~Gy}$, and thereafter only the PF to 70 Gy. At least $95 \%$ of the PTV received the prescribed doses. Less than $15 \%$ of the outlined rectal volume received doses $>70$ Gy. All patients were given 3D conformal therapy with high-energy linear accelerators equipped with multi-leaf collimators, and they were treated in the supine position. The external beam radiation was performed with a four-field at Radiumhemmet, whereas a three-field technique (one anterior and two lateral fields) was used at Södersjukhuset; all fields were equally weighted at both facilities.

A median of 3 months of NHT was given to 165 (90\%) of the patients. Of these, $151(92 \%)$ received antiandrogens (bicalutamide $50 \mathrm{mg}$ x 1 or flutamide $250 \mathrm{mg}$ x 3) combined with a gonadotropin-releasing hormone $(\mathrm{GnRH})$ analogue, and $14(8 \%)$ were given antiandrogens or $\mathrm{GnRH}$ analogue as monotherapy.

Data collection. Clinical data were collected from electronic medical records (KS database). Side-effects were reported according to the GU-GI toxicity scale of the Radiation Therapy Oncology Group (RTOG) and the European Organization for Research and Treatment of Cancer (EORTC) (11). The Charlson co-morbidity index adjusted for age was analyzed to correlate co-morbidities and RTOG toxicity $(12,13)$. DRE was performed during the regular follow-up after RP. A palpable post-RP recurrence was found in $46(25 \%)$ patients; $35(76 \%)$ of these underwent transrectal ultrasound guided fine-needle aspiration cytology. Pathological evidence of local post-RP recurrence was found in 10 (29\%). The patient characteristics are described in Table I. The investigation was approved by the local ethics committee (approval number 2006/620-31/1).

RTOG score and Charlson co-morbidity index adjusted for age (CCIAA) (Table II)

HRQoL EORTC QLQ-C30 and PR-25 (prostate specific) $(14,15)$. Up to October 2009,9 of the 184 consecutive patients had died during follow-up. To each of the remaining 175, an envelope containing both questionnaires, a letter inviting participation in the study, and a prepaid return envelope was sent. In all, $148(85 \%)$ patients returned the questionnaires; four were blank, which left 144 (83\%) for evaluation.

Statistical analysis. Pre-SRT BcF was defined as follows: a rise in PSA $>0.1 \mathrm{ng} / \mathrm{dl}$ when nadir was undetectable, or any rise in PSA above nadir. Clinical characteristics such as pre-RP PSA and pT stage (extracapsular extension), pre-RT PSADT, post-RP GS, pre-SRT PSA, were dichotomized. Post-SRT BcF was defined as any detectable PSA with an undetectable nadir or an elevation of $0.1 \mathrm{ng} / \mathrm{ml}$ above nadir. Undetectable PSA 

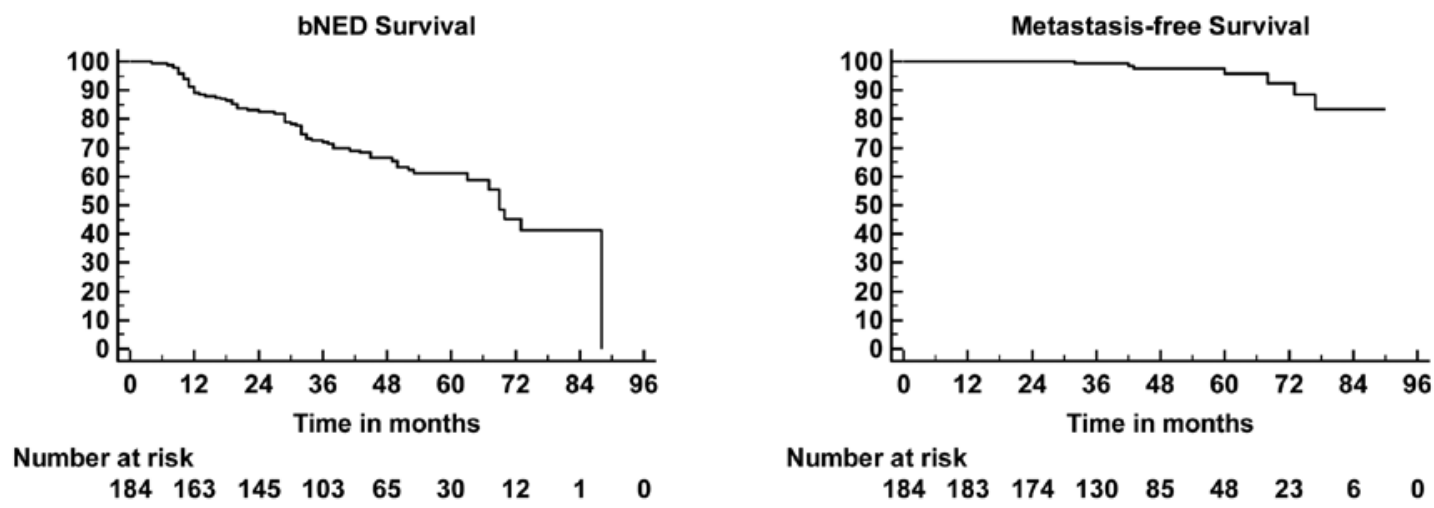

Figure 1. Metastasis-free and bNED survival curves. Patients with BcF had a median bNED period of 30 months (4-88).

was reported as $<0.05 \mathrm{ng} / \mathrm{ml}$, and an approximation to $0.04 \mathrm{ng}$ / $\mathrm{ml}$ was done in the statistical calculation. The PSADT calculation was done according to Pound et al (1) The pT stage was defined according to the 1997 TNM classification system.

No biochemical evidence of disease (bNED), overall survival (OS), PCa-specific survival, and metastasis-free survival (MFS) were estimated by using the Kaplan-Meier and log-rank methods to demonstrate differences. Time to event was calculated from the date of SRT to the date of the event or death. A univariate regression was performed in which $\mathrm{BcF}$ was evaluated as an outcome variable. The multivariate regressions were conducted with stepwise variable elimination, using $\mathrm{BcF}$ and bone metastases as outcome variables. Both univariate and multivariate analyses were performed using Cox proportional hazards regression and were compared with the results presented by Stephenson et al (16) as listed in Table III. Logarithmic transformation of the pre-SRT PSA was performed to run a logistic regression.

The raw scores of the questionnaires were linearly transformed into a 100-point scale, and missing values were imputed according to the EORTC scoring manual (17). Mean questionnaire scores for patients with $\mathrm{bNED}$ and with $\mathrm{BcF}$ were calculated with $95 \%$ confidence intervals.

To compare means, the Student's t-test was used when data were normally distributed, and the Mann-Whitney U test when skewed. Fisher's exact test was utilized for the count/frequency analyses. The tests were performed using STATA 9, and p-values $<0.05$ were considered statistically significant.

Using the nomogram. The pre-treatment data of patients were used in the nomogram for radiotherapy as described by Stephenson et al (7) to create an individual probability for PSA bNED at 3 years. Thereafter, the means were calculated.

\section{Results}

Nine patients died during follow-up and one of PCa. This gave a $99 \%$ PCa-specific survival and $95 \%$ OS. Seven (4\%) developed bone metastases. No statistically significant differences in OS were found between patients with low/medium and high CCIAA scores. Using the patients' pre-treatment data and the Stephenson nomogram, a mean 3-year bNED probability of $53 \%$ (95\% CI 49.21; 56.71) was calculated.

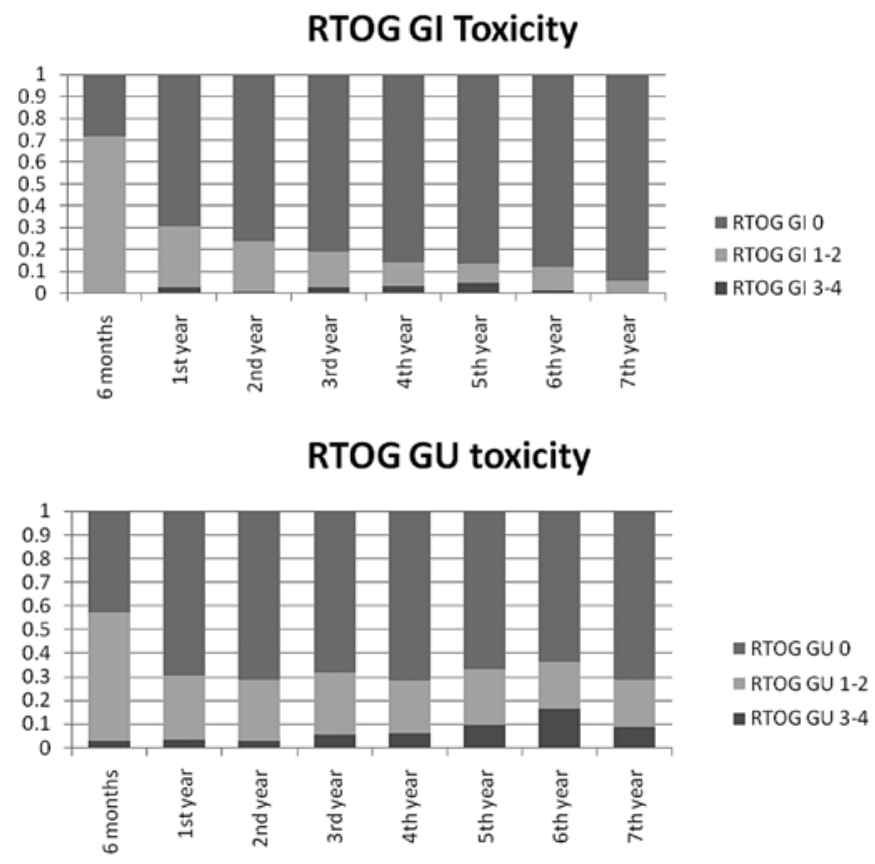

Figure 2. Gastrointestinal (GI) and genitourinary (GU) RTOG toxicity after $\leq 7$ years of follow-up.

PSA levels after SRT + NHT were undetectable in $171(93 \%)$ patients. BcF developed in 68 (37\%). Kaplan-Meier bNED, MFS, curves are shown in Fig. 1.

In the univariate analyses, post-RP GS, pre-SRT PSA, pT stage, SVI, and detectable post-SRT PSA were statistically significant prognostic factors for $\mathrm{BcF}$. In the multivariate analyses for $\mathrm{BcF}$, statistical significance was found for the same variables as in the univariate analysis, except for pT stage. In the multivariate analysis for metastases, only SVI was found to be significant (Table III). After a logistic regression, pre-SRT PSA was a positive prognostic factor for $\mathrm{BcF}(\mathrm{p}<0.001$, OR $5.4895 \%$ CI 2.27; 13.23). The area under the curve was 0.68 (95\% CI 0.61; 0.75), and the z-test statistic 4.30 (p<0.001).

Grade 1-2 acute toxicities were observed in the GU and the GI region in $100(54 \%)$ and $132(72 \%)$ patients, respectively, and the former proportion, tended to diminish over time (Fig. 2). Corresponding proportions showing RTOG acute toxicity grade 3-4 were observed $5(3 \%)$ and $0(0 \%)$ patients, respectively. 


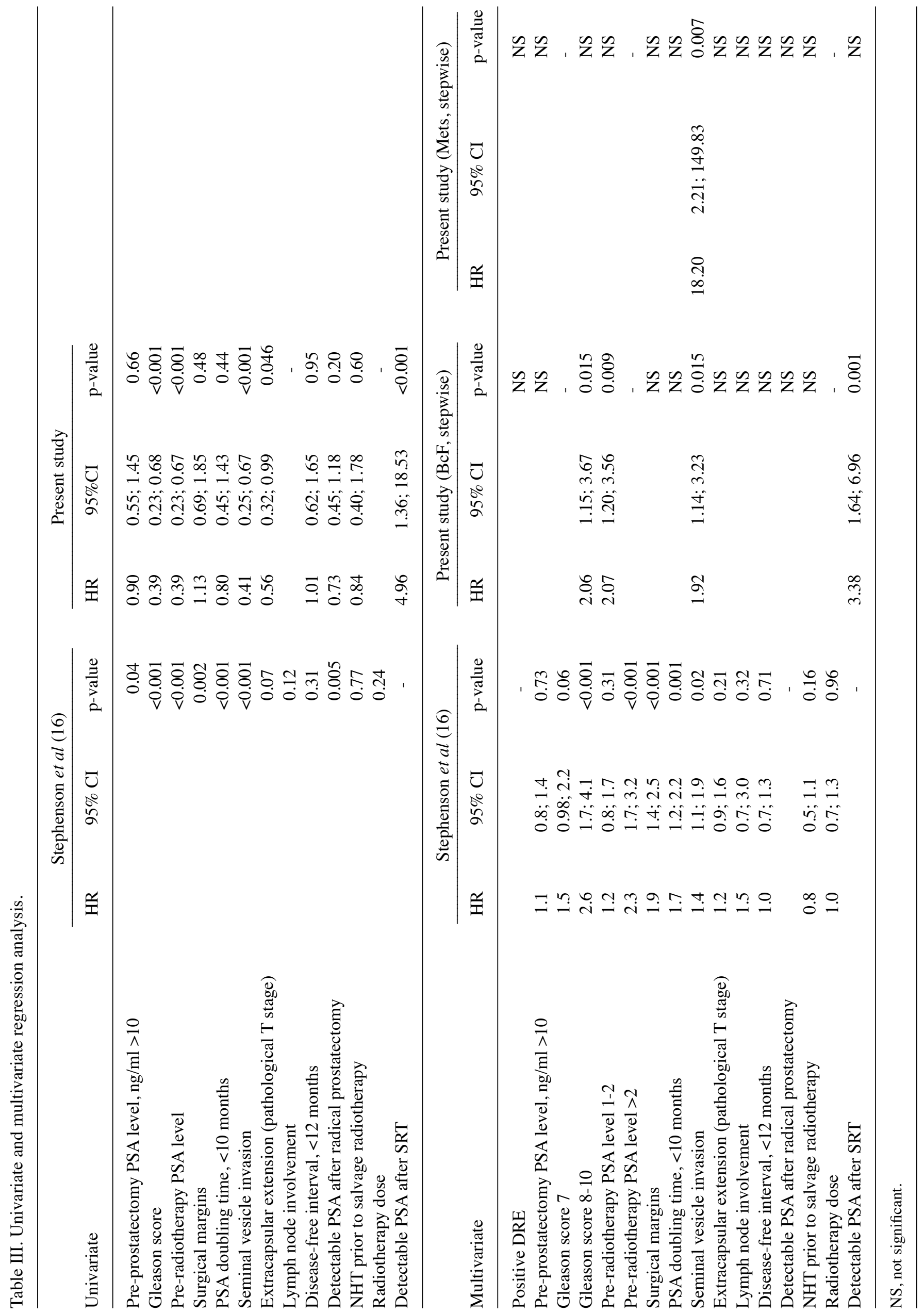




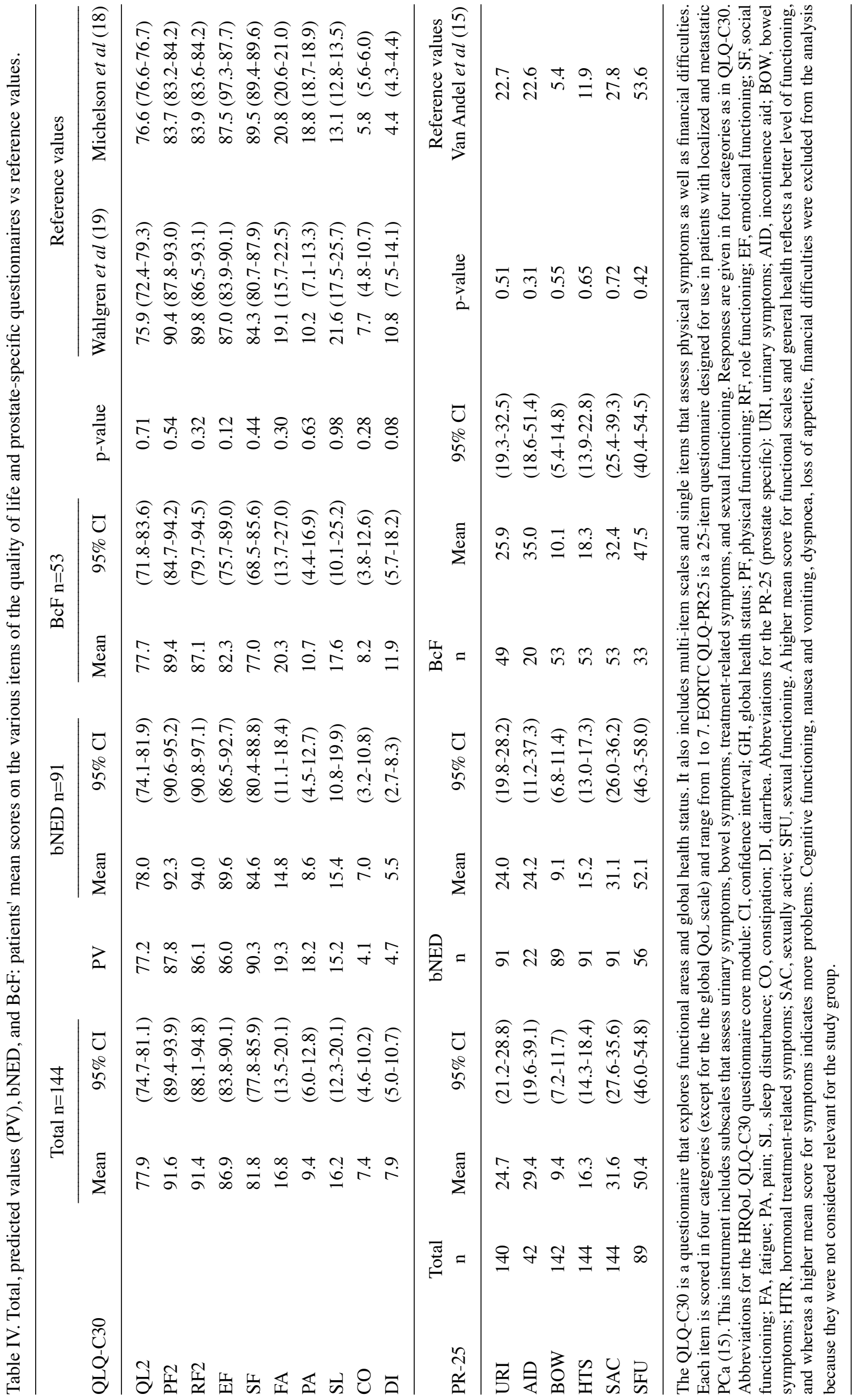


Table V. Proportion of patients with poor prognostic factors who still had undetectable PSA values after SRT.

\begin{tabular}{|c|c|c|c|c|c|c|}
\hline & $\mathrm{DRE}+$ & Biopsy + & $\mathrm{SV}+$ & Gleason 8-10 & pre-SRT PSA >1 & PSADT $<10$ \\
\hline Total n & 46 & 10 & 40 & 29 & 31 & 134 \\
\hline $\begin{array}{l}\text { Undetectable PSA, n (\%) } \\
\text { Median follow-up (months) }\end{array}$ & $25(54.3)$ & $6(60)$ & $17(42.5)$ & $13(44.8)$ & $11(35.5)$ & 80 (59.7) \\
\hline $\begin{array}{l}\text { Total (range) } \\
\text { Median follow-up (months) }\end{array}$ & $50(12-92)$ & $41(30-83)$ & $54(28-92)$ & $42(13-90)$ & $46(24-92)$ & $50(1-92)$ \\
\hline $\begin{array}{l}\text { Patients with undetectable } \\
\text { PSA (range) }\end{array}$ & $44(12-79)$ & $38(30-42)$ & $55(17-83)$ & $42(13-83)$ & $44(41-64)$ & $49(13-84)$ \\
\hline
\end{tabular}

An increment in the frequency of grade over time was observed for both GU and GI toxicity, although it was more evident for the former. At the 2- and 5-year follow-ups, respectively, the numbers of patients with grade 3-4 toxicities were $1(1 \%)$ and $5(5 \%)$ as regards the GI tract, and $5(3 \%)$ and $11(9 \%)$ as regards the GU region.

The CCIAA score was low/medium for $115(63 \%)$ patients and high for 69 (38\%). No statistically significant association was found between RTOG toxicity and CCIAA scores (Table II).

In Table IV, the results for each domain of the two questionnaires are shown together with predicted values for the general population in Sweden and values obtained in a Swedish longterm follow-up of men with localized prostate cancer $\geq 5$ years after brachytherapy $(18,19)$.

\section{Discussion}

One of the aims of the present study was to identify prognostic variables for patients who may derive long-term benefit from SRT at a dose of 70 Gy combined with short-term NHT. Stephenson et al (16) analyzed retrospectively the outcome of 501 patients who were treated with SRT at five different academic centres with a median follow-up of 45 months. These patients had received a heterogeneous pelvic radiation dose (PRD) with a mean absorbed dose of 64.8 Gy. In that study, GS 8-10, pre-SRT PSA $>2.0 \mathrm{ng} / \mathrm{ml}$, positive surgical margins, PSADT $\leq 10$ months, and SVI were all significant predictors of disease progression. In the present study, 184 consecutive patients were included in the study and treated with SRT at a single institution with a homogeneous PRD and NHT (median 3 months). The median follow-up time was 48 months. None of the patients were lost to follow-up. Our results show that the significant factors for treatment failure were post-RP GS, pre-SRT PSA $\geq 1.0 \mathrm{ng} / \mathrm{ml}, \mathrm{SVI}$, and detectable post-SRT PSA, the latter in accordance with data presented by Wiegel et al (5). We interpret these findings as indicating that the importance of some of the poor prognostic factors identified by Stephenson and colleagues can be diminished by using higher SRT doses combined with short-term NHT.

Once post-RP BcF has been diagnosed, SRT remains the only potentially curative therapy (16). For SRT, the American Society for Therapeutic Radiology and Oncology consensus guidelines recommend the use of the highest dose of radiation that can be delivered with acceptable morbidity, and suggest a minimum of 64 Gy with conventional fractionation
(20). However, doses $>66.6$ Gy have been described to result in decreased risk of $\mathrm{BcF}$ (21). With the advent of conformal radiotherapy techniques, the maximally tolerated and effective PRD for recurrent PCa is still unclear (22) and late toxicity, especially rectal, is the dose-limiting factor.

MacDonald et al (23) and Choo et al (24) have reported that PCa patients with palpable recurrence after RP respond poorly to SRT. In our study, this was not statistically significant as an independent prognostic factor and no difference between the patients with positive and negative pathological disease was detected as regards the risk of $\mathrm{BcF}$ or metastasis. However, this is difficult to compare, since the PRD used in previous studies have been lower and, in many instances, heterogeneous in terms of dosing and fractionation. Also, some other previous studies have indicated that routine biopsies taken at the anastomotic site before treatment are not advisable, since a negative biopsy does not exclude local recurrence, and a positive biopsy does not exclude systemic disease $(21,25)$. Thus, it remains to be determined whether a negative or positive biopsy, with no evidence of metastatic disease, would change the treatment approach used in patients with BcF after RP. Nevertheless, in the present study, the PRD may have had an impact on DRE as a prognostic factor. Caution must be taken when interpreting these results due the following: the numbers of patients with positive and negative, respectively, DRE differed and both groups showed statistically significant differences in clinical characteristics (data not shown).

The current results demonstrate that a substantial proportion of patients in our study achieved a durable response in terms of bNED, despite having a high GS, advanced pT stage, positive DRE, positive surgical margins, rapid PSADT, and SVI, all of which have been considered to be incurable (Table V). In addition, our observations indicate $63 \%$ bNED (99\% of those with still undetectable PSA), 99\% PCa-specific survival, 95\% OS, and $96 \%$ MFS at 4-year (median) follow-up. These findings are in contrast to the 4-year progression-free probability of $45 \%$ reported by Stephenson et al. The data concerning MFS in the present study have to be interpreted with caution since radiologic follow-up was not performed systematically and due to the fact that some patients actively asked for hormone therapy although metastatic disease could not be verified radiologically.

In a study by Tzou et al (26), mean radiation doses of 60-70 and $\geq 70$ Gy were found to be associated with bNED in $25-57 \%$ and $58-67 \%$ of cases, respectively. The definition of BcF after $\mathrm{RP}$ has been a matter of debate. The American Urological 
Association and the European Association of Urology designate it as a PSA level of $0.2 \mathrm{ng} / \mathrm{ml}$ or higher, with a second confirmatory value of $>0.2 \mathrm{ng} / \mathrm{ml}$ (27-29). The use of a too low PSA threshold for SRT involves the risk of over-treating patients. Some investigators have described that $\leq 50 \%$ of patients with a single PSA elevation $<0.4 \mathrm{ng} / \mathrm{ml}$ after RP may be regarded as having stable non-progressive disease. This is based on the assumption that residual benign prostatic tissue might be responsible for the rise in PSA $(16,30)$. However, recent data suggest that residual benign prostatic elements after RP are an unlikely source of elevated PSA (31), a finding which has led to a decision to administer early SRT at our institute.

The nomogram presented by Stephenson et al was used in the present study to compare the actual and the expected outcome of SRT. A substantially higher bNED was found in the present study, $63 \%$ at 4 years, compared with $53 \%$ at 3 years when using the Stephenson nomogram. The reasons for the improved outcome in our series may be explained by the use of a higher SRT total dose combined with the use of short-term NHT. However, there may also exist other plausible explanations for the discrepancies seen.

Stephenson et al (32) stated that the potential for morbidity associated with RT argues against indiscriminate use of this treatment in the salvage setting. The incidence of RTOG GI/GU grade 3-4 acute toxicity has been reported to be $<4 \%$ (3,33-37), which is in agreement with the results obtained in the present series. GI/ GU grade 1-2 and grade 3-4 late toxicities have been reported to occur in 5-20\% and $<4 \%$ of SRT patients, respectively (33-36,38). We observed GU/GI late toxicities as grade 1-2 in 23 and $9 \%$, respectively, and as grade 3-4 in 9 and 5\%, respectively. This slight increase in GU/GI complications is most probably doserelated. It may, thus, be possible to reduce the frequency of these toxicities further by the use of modern techniques such as intensity-modulated radiation therapy (IMRT) (39). Interestingly, the incidence of toxicity was not more pronounced in patients with a high, as compared with low/intermediate, CCIAA score. The occurrence of co-morbidity often influences the choice of treatment and it is generally agreed that men with severe comorbidities receive less aggressive treatment (40). To our knowledge, the present study is the first to describe the co-morbidity pattern and the influence of this in patients undergoing treatment with 70 Gy SRT + NHT.

The data presented in this report support the idea that patients with a rise in PSA after RP should be offered 70 Gy SRT with a curative intention. Although there are numerous reports concerning SRT, ours is one of the largest single-institution reports describing treatment using a high and homogeneous PRD + NHT, given to consecutive patients for a delimited period of time during which no major alterations were done in treatment policies. Androgen deprivation before radiotherapy has shown improvement in DSS and OS in patients with locally advanced and high risk tumours (41). The role for NHT in combination with SRT still unclear although recent data from other treatment series have, in addition to ours, shown promising results in patients undergoing SRT $(42,43)$. The role for NHT in combination with SRT is currently being addressed in ongoing randomized studies.

The patients in the current investigation had slightly better scores on almost all items of the HRQoL (EORTC QLQ-C30) after 4-year follow-up compared with predicted values from an age-matched healthy population and the results presented by Wahlgren et al and Michelson et al $(18,19)$. Surprisingly, sexual functioning was not poorer in patients evaluated in the present series. No statistical differences were found between patients with or without $\mathrm{BcF}$, although some complications, such as diarrhoea, may be clinically relevant. The PR-25 results in our study show slightly higher negative impact than those presented by van Andel et al (15), a finding that was also more or less anticipated. To our knowledge, the present study is one of the largest that assesses long-term HRQoL in patients treated with SRT.

In conclusion, salvage radiotherapy with 70 Gy delivered in fractions of $2 \mathrm{~Gy} /$ day combined with a 3-month NHT results in long-term undetectable PSA in more than half of patients presenting with biochemical and/or clinical recurrence after radical prostatectomy.

The data obtained in the present study show that nonmetastatic patients should not be routinely disqualified from receiving SRT although per se presenting with poor prognostic factors at surgery such as SVI and/or high GS or having a pre-SRT PSA level $>1.0 \mathrm{ng} / \mathrm{ml}$ and/or a short pre-SRT PSADT. SRT combined with short-term NHT is associated with acceptable rectal-genitourinary toxicity. No obvious association was found between the occurrence of co-morbidity (assessed with CCIAA) and acute/long-term toxicities. The treatment can be safely delivered without any major negative effects on long-term QoL.

\section{Acknowledgements}

We would like to acknowledge Dr Hemming Johansson for biostatistical expertise. This study was supported by the Swedish Cancer Society, the National Council of Science and Technology (Consejo Nacional de Ciencia y Tecnologia, CONACyT), UANL, Radiumhemmets Research Funds, and The Af Jochnick Research Foundation.

\section{References}

1. Pound CR, Partin AW, Eisenberger MA, Chan DW, Pearson JD and Walsh PC: Natural history of progression after PSA elevation following radical prostatectomy. JAMA 281: 15911597, 1999.

2. Ward JF, Blute ML, Slezak J, Bergstralh EJ and Zincke H: The long-term clinical impact of biochemical recurrence of prostate cancer 5 or more years after radical prostatectomy. J Urol 170: 1872-1876, 2003.

3. Bolla M, van Poppel H, Collette L, et al: Postoperative radiotherapy after radical prostatectomy: a randomised controlled trial (EORTC trial 22911). Lancet 366: 572-578, 2005.

4. Thompson IM, Tangen CM, Paradelo J, et al: Adjuvant radiotherapy for pathological T3NOM0 prostate cancer significantly reduces risk of metastases and improves survival: long-term follow-up of a randomized clinical trial. J Urol 181: 956-962, 2009.

5. Wiegel T, Lohm G, Bottke D, et al: Achieving an undetectable PSA after radiotherapy for biochemical progression after radical prostatectomy is an independent predictor of biochemical outcome - results of a retrospective study. Int J Radiat Oncol Biol Phys 73: 1009-1016, 2009.

6. Buskirk SJ, Pisansky TM, Schild SE, et al: Salvage radiotherapy for isolated prostate specific antigen increase after radical prostatectomy: evaluation of prognostic factors and creation of a prognostic scoring system. J Urol 176: 985-990, 2006.

7. Stephenson AJ and Slawin KM: The value of radiotherapy in treating recurrent prostate cancer after radical prostatectomy. Nat Clin Pract Urol 1: 90-96, 2004. 
8. Moreira DM, Banez LL,Presti JC Jr, et al: Predictors of secondary treatment following biochemical recurrence after radical prostatectomy: results from the Shared Equal Access Regional Cancer Hospital database. BJU Int 105: 28-33, 2010.

9. Ost P, De Troyer B, Fonteyne V, Oosterlinck W and De Meerleer G: A matched control analysis of adjuvant and salvage high-dose postoperative intensity-modulated radiotherapy for prostate cancer. Int J Radiat Oncol Biol Phys 80: 1316-1322, 2011.

10. Trabulsi EJ, Valicenti RK, Hanlon AL, et al: A multi-institutional matched-control analysis of adjuvant and salvage postoperative radiation therapy for pT3-4N0 prostate cancer. Urology 72: 1298-1304, 2008.

11. Cox JD, Stetz J and Pajak TF: Toxicity criteria of the Radiation Therapy Oncology Group (RTOG) and the European Organization for Research and Treatment of Cancer (EORTC). Int J Radiat Oncol Biol Phys 31: 1341-1346, 1995.

12. Charlson ME, Pompei P, Ales KL and MacKenzie CR: A new method of classifying prognostic comorbidity in longitudinal studies: development and validation. J Chronic Dis 40: 373-383, 1987.

13. Extermann M: Measuring comorbidity in older cancer patients. Eur J Cancer 36: 453-471, 2000.

14. Aaronson NK, Ahmedzai S, Bergman B, et al: The European Organization for Research and Treatment of Cancer QLQ-C30: a quality-of-life instrument for use in international clinical trials in oncology. J Natl Cancer Inst 85: 365-376, 1993.

15. van Andel G, Bottomley A, Fossa SD, et al: An international field study of the EORTC QLQ-PR25: a questionnaire for assessing the health-related quality of life of patients with prostate cancer. Eur J Cancer 44: 2418-2424, 2008.

16. Stephenson AJ, Shariat SF, Zelefsky MJ, et al: Salvage radiotherapy for recurrent prostate cancer after radical prostatectomy. JAMA 291: 1325-1332, 2004

17. Fayers P, Aaronson N, Bjordal K, Groenvold M, Curran D and Bottomley A: EORTC QLQ-C30 scoring manual. European Organization for Research and Treatment of Cancer. 3rd edition. 2001.

18. Michelson $\mathrm{H}$, Bolund $\mathrm{C}$, Nilsson $\mathrm{B}$ and Brandberg $\mathrm{Y}$ : Health-related quality of life measured by the EORTC QLQ-C30reference values from a large sample of Swedish population. Acta Oncol 39: 477-484, 2000.

19. Wahlgren T, Nilsson S, Lennernas B and Brandberg Y: Promising long-term health-related quality of life after high-dose-rate brachytherapy boost for localized prostate cancer. Int J Radiat Oncol Biol Phys 69: 662-670, 2007.

20. Cox JD, Gallagher MJ, Hammond EH, Kaplan RS and Schellhammer PF: Consensus statements on radiation therapy of prostate cancer: guidelines for prostate re-biopsy after radiation and for radiation therapy with rising prostate-specific antigen levels after radical prostatectomy. American Society for Therapeutic Radiology and Oncology Consensus Panel. J Clin Oncol 17: 1155, 1999.

21. Bernard JR, Jr., Buskirk SJ, Heckman MG, et al: Salvage radiotherapy for rising prostate-specific antigen levels after radical prostatectomy for prostate cancer: dose-response analysis. Int J Radiat Oncol Biol Phys 76: 735-740, 2010.

22. Kupelian PA, Willoughby TR, Reddy CA, Klein EA and Mahadevan A: Hypofractionated intensity-modulated radiotherapy (70 Gy at 2.5 Gy per fraction) for localized prostate cancer: Cleveland Clinic experience. Int J Radiat Oncol Biol Phys 68: 1424-1430, 2007.

23. MacDonald OK, Schild SE, Vora S, et al: Salvage radiotherapy for men with isolated rising PSA or locally palpable recurrence after radical prostatectomy: do outcomes differ? Urology 64: 760-764, 2004

24. Choo R, Morton G, Danjoux C, Hong E, Szumacher E and DeBoer G: Limited efficacy of salvage radiotherapy for biopsy confirmed or clinically palpable local recurrence of prostate carcinoma after surgery. Radiother Oncol 74: 163-167, 2005.
25. Neulander EZ and Soloway MS: Failure after radical prostatectomy. Urology 61: 30-36, 2003.

26. Tzou K, Tan WW and Buskirk S: Treatment of men with rising prostate-specific antigen levels following radical prostatectomy. Expert Rev Anticancer Ther 11: 125-136, 2011.

27. Boccon-Gibod L, Djavan WB, Hammerer P, et al: Management of prostate-specific antigen relapse in prostate cancer: a European Consensus. Int J Clin Pract 58: 382-390, 2004.

28. Cookson MS, Aus G, Burnett AL, et al: Variation in the definition of biochemical recurrence in patients treated for localized prostate cancer: the American Urological Association Prostate Guidelines for Localized Prostate Cancer Update Panel report and recommendations for a standard in the reporting of surgical outcomes. J Urol 177: 540-545, 2007.

29. Heidenreich A, Aus G, Bolla M, et al: EAU guidelines on prostate cancer. Eur Urol 53: 68-80, 2008.

30. Amling CL, Bergstralh EJ, Blute ML, Slezak JM and Zincke H: Defining prostate specific antigen progression after radical prostatectomy: what is the most appropriate cut point? J Urol 165: 1146-1151, 2001

31. Godoy G, Tareen BU and Lepor H: Does benign prostatic tissue contribute to measurable PSA levels after radical prostatectomy? Urology 74: 167-170, 2009.

32. Stephenson AJ, Scardino PT, Kattan MW, et al: Predicting the outcome of salvage radiation therapy for recurrent prostate cancer after radical prostatectomy. J Clin Oncol 25: 2035-2041, 2007.

33. Catton C, Gospodarowicz M, Warde P, et al: Adjuvant and salvage radiation therapy after radical prostatectomy for adenocarcinoma of the prostate. Radiother Oncol 59: 51-60, 2001.

34. Katz MS, Zelefsky MJ, Venkatraman ES, Fuks Z, Hummer A and Leibel SA: Predictors of biochemical outcome with salvage conformal radiotherapy after radical prostatectomy for prostate cancer. J Clin Oncol 21: 483-489, 2003.

35. Maier J, Forman J, Tekyi-Mensah S, Bolton S, Patel R and Pontes JE: Salvage radiation for a rising PSA following radical prostatectomy. Urol Oncol 22: 50-56, 2004.

36. Pacholke HD, Wajsman Z, Algood CB, Morris CG and Zlotecki RA: Postoperative adjuvant and salvage radiotherapy for prostate cancer: impact on freedom from biochemical relapse and survival. Urology 64: 982-986, 2004.

37. Valicenti RK, Gomella LG, Ismail M, et al: Durable efficacy of early postoperative radiation therapy for high-risk pT3N0 prostate cancer: the importance of radiation dose. Urology 52: 1034-1040, 1998

38. Anscher MS, Clough R and Dodge R: Radiotherapy for a rising prostate-specific antigen after radical prostatectomy: the first 10 years. Int J Radiat Oncol Biol Phys 48: 369-375, 2000

39. Alongi F, Fiorino C, Cozzarini $\mathrm{C}$, et al: IMRT significantly reduces acute toxicity of whole-pelvis irradiation in patients treated with post-operative adjuvant or salvage radiotherapy after radical prostatectomy. Radiother Oncol 93: 207-212, 2009.

40. Berglund A, Garmo H, Tishelman C, Holmberg L, Stattin P and Lambe M: Comorbidity, treatment and mortality: a population based cohort study of prostate cancer in PCBaSe Sweden. J Urol 185: 833-840, 2011.

41. Widmark A, Klepp O, Solberg A, et al: Endocrine treatment, with or without radiotherapy, in locally advanced prostate cancer (SPCG-7/SFUO-3): an open randomised phase III trial. Lancet 373: 301-308, 2009.

42. Choo R, Danjoux C, Gardner S, et al: Efficacy of salvage radiotherapy plus 2-year androgen suppression for postradical prostatectomy patients with PSA relapse. Int J Radiat Oncol Biol Phys 75: 983-989, 2009.

43. Pai HH, Eldridge B, Bishop D, et al: Does neoadjuvanthormone therapy improve outcome in prostate cancer patients receiving radiotherapy after radical prostatectomy? Can J Urol 16: 4541-4552, 2009. 